\title{
Exploración del pié a los pacientes diabéticos de una unidad de hemodiálisis
}

\author{
Eloísa Bernal Pedreño - Esther Salces Sáez - Antonia Sambruno Giráldez
}

Unidad de cuidados nefrológicos - Hospital Universitario de Puerto Real

\section{Resumen}

El Plan Andaluz de Salud y el Plan Integral de Diabetes de Andalucía cuentan entre sus objetivos reducir las amputaciones y mejorar la atención a los pacientes afectos de esta patología. Una prevención adecuada y un correcto tratamiento pueden reducir las amputaciones hasta un $85 \%$. Nuestro objetivo fue evaluar la prevalencia del "Riesgo de lesión relacionado con disfunción sensorial e hipoxia tisular" y realizar una clasificación por riesgo, para un mejor seguimiento y prevención de ulceras y amputaciones.

Realizamos un estudio descriptivo y transversal sobre los pacientes diabéticos en hemodiálisis para clasificarlos según riesgo, siguiendo la guía de manejo de diabetes basada en la evidencia del Scottish Intercollegiate Guidelines Network.

En el 77,4\% de los pacientes nunca se había valorado el riesgo de pie diabético. El 34,48 de los pacientes presentaba un riesgo alto de lesión, el $34,48 \%$ riesgo moderado y un 22,58 bajo riesgo. Un 6,45\% presentaba pie diabético.

La ausencia de exploración anterior en la mayoría de los pacientes muestra un seguimiento inadecuado. La alta prevalencia de riesgo de lesión hace necesario un seguimiento protocolizado.

\begin{tabular}{|c|}
\hline Correspondencia: \\
Eloísa Bernal Pedreño \\
Unidad de cuidados nefrológicos \\
Hospital Universitario de Puerto Real \\
Carretera N. IV Km. 665 \\
11510. Puerto Real. Cádiz \\
eloisabernal@gmail.com \\
\hline
\end{tabular}

\section{PALABRAS CLAVE:}

- HEMODIÁLISIS

- PIE DIABÉTICO

- PREVENCIÓN DE AMPUTACIONES

Examination of the foot of diabetic patients in a haemodialysis unit

\section{Abstract}

The objectives of the Andalusian Health Plan and the Integral Diabetes Plan of Andalusia include a reduction in amputations and an improvement in care for patients with this disease. Adequate prevention and correct treatment can reduce amputations by up to $85 \%$. Our goal was to evaluate the prevalence of the "Risk of injury related to sensorial dysfunction and tissue hypoxy" and carry out a risk-based classification, for better monitoring and prevention of ulcers and amputations.

We carried out a descriptive, transversal study on diabetic patients undergoing haemodialysis to classify them according to the risk, following the evidence-based diabetes management guide of the Scottish Intercollegiate Guidelines Network.

In $77.4 \%$ of patients, the risk of diabetic foot had never been assessed. Of the patients, 34.48 presented a high risk of injury, $34.48 \%$ moderate risk and 22.58 low risk. $6.45 \%$ presented diabetic foot.

The absence of any prior examination in most patients shows inadequate monitoring. The high prevalence of risk of injury shows the need for protocolized monitoring. 


\section{KEY WORDS:}

- HAEMODIALYSIS

- DIABETIC FOOT

- PREVENTION OF AMPUTATIONS

\section{Introducción}

La diabetes es un importante problema sanitario tanto a nivel individual como de salud públical ${ }^{1}$. Supone además entre el $20-30 \%$ de las causas de inclusión en programas de diálisis en España².

Para la Sociedad Española de Angiología y Cirugía Vascular, el Pie Diabético se define como "Alteración clínica de base etiopatogénica neuropática inducida por la hiperglucemia mantenida, en la que, con o sin la existencia de isquemia y previo desencadenante traumático, produce lesión y/o ulceración del pie". Afecta al $15 \%$ de los diabéticos, origina frecuentes ingresos hospitalarios y puede terminar con la pérdida de la extremidad 3 . Entre el $40 \%$ y el $60 \%$ de las amputaciones no traumáticas de la extremidad inferior ocurren en pacientes diabéticos y el $85 \%$ de estas amputaciones viene precedido de una úlcera en el pie $e^{4}$.

El Plan Andaluz de Salud y el Plan Integral de Diabetes de Andalucía cuentan entre sus objetivos reducir estas amputaciones y adecuar la atención a las necesidades de la población de manera efectiva y eficiente ${ }^{2}$. Se ha demostrado que una prevención adecuada de las lesiones en el pie del diabético, así como un correcto tratamiento puede reducir esta tasa de amputación entre un 50 y un $85 \%{ }^{4}$.

Dadas las incapacidades, las repercusiones sociales y el elevado coste económico que origina la pérdida de la extremidad, se han propuesto diversos documentos de consenso para la creación de equipos multidisciplinares expertos e interesados en este tipo de patología, que sepan reconocer los factores de riesgo relacionados con la aparición de las lesiones, así como, desarrollar estrategias preventivas y de tratamiento precoz ${ }^{4}$. Es claro también que para la prevención del pie diabético, la detección de problemas y su corrección es necesario promover la educación del paciente y el autocuidado $0^{4,5}$.
La exploración del pie del diabético debe constar de inspección visual y hábitos higiénicos, exploración neuropática y valoración de la capacidad de autocuidado ${ }^{4,6}$. La exploración con diapasón de $128 \mathrm{~Hz}$. y monofilamento de Semmes-Weinstein es un método adecuado como screening en la neuropatía diabéti-

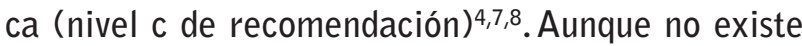
evidencia firme sobre la frecuencia en que estas revisiones deben hacerse ${ }^{7}$, se acepta la realización de al menos un examen anual de los pies en las personas con diabetes y con mayor frecuencia si existen factores de riesgo que así lo indiquen $n^{4,7,9,10}$.

Encontramos además que la diabetes es uno de los factores que más se asocia a la arteriopatía periférica en los pacientes que reciben diálisis como describen Lancho y colaboradores. ${ }^{11,12}$

Son muchos los estudios que podemos encontrar sobre pacientes diabéticos en tratamiento de hemodiálisis. Tanto Lipscombe como Pliakogiannis encontraron que un programa de prevención llevado por podólogos podría ser útil en la prevención de amputaciones en pacientes diabéticos en programa de diálisis peritoneal ${ }^{13,14}$. La mayoría de los autores sin embargo defienden un enfoque multidisciplinar del problema donde el papel de la enfermera es necesario ${ }^{15}$.

Locking-Cusolito y colaboradores, movidos por la alta prevalencia de amputaciones en los pacientes en hemodiálisis analizan los factores de riesgo, así como la capacidad de autocuidado de dichos pacientes y concluyen en la necesidad de valoraciones, educación para el auto-cuidado y la derivación al especialista si fuese necesario ${ }^{16}$.

Sin embargo, a pesar del papel fundamental de la enfermería, las intervenciones enfermeras sobre prevención de las amputaciones y su registro son descritos también como objetivos pendientes en los planes de cuidados de estos pacientes. ${ }^{11,12}$

Podemos encontrar estudios que reflejan también el beneficio de la aplicación de las estrategias de prevención de amputaciones para la población general de diabéticos en los pacientes que reciben tratamiento de hemodiálisis. 
Speckman nos habla de la influencia de la diabetes en la amputación de miembros inferiores de pacientes en hemodiálisis y de los beneficios de la adecuación de la dosis de diálisis, del tratamiento de las comorbilidades cardiovasculares y de las estrategias de prevención de amputaciones ${ }^{17}$. McMurray en su estudio "Diabetes education and care management significantly improve patient outcomes in the dialysis unit" encuentra resultados estadísticamente significativos en el numero de amputaciones, en cifras de glicemia y en la calidad de vida tras realizar un programa educativo en diabetes, de tal forma que la Asociación de Diabetes Americana (ADA) reconoció las unidades de diálisis como centros apropiados para proporcionar educación en diabetes ${ }^{18}$ Aunque también encontramos otros en los que se recomienda en estas situaciones no exagerar la importancia de la prevención y sí el papel del fósforo ${ }^{19}$.

Nos planteamos pues los siguientes objetivos:

- Evaluar la prevalencia del diagnóstico "Riesgo de lesión relacionado con disfunción sensorial e hipoxia tisular" 20 de los pacientes con diabetes en programa de hemodiálisis en nuestro centro, en relación con el síndrome pie diabético, dentro del marco de una valoración y atención integral de enfermería.

- Realizar una clasificación por riesgo basada en la evidencia existente, que permita un mejor seguimiento y prevención de las ulceras y amputaciones en miembros inferiores.

\section{Población y Métodos}

Se realizó un estudio descriptivo y transversal entre febrero y marzo del 2008, sobre la totalidad de la población de pacientes diabéticos con IRCT en programa de hemodiálisis del Hospital Universitario de Puerto Real, resultando un total de 36 pacientes. Se excluyeron del estudio a los pacientes que expresaron su negativa a ser explorados (dos) y a los que, por deterioro cognitivo importante, no pudieran responder con fiabilidad a los cuestionarios y pruebas realizadas (dos).

Entre la información recogida se encuentran:

- Datos personales, factores de riesgo, lesiones previas, prácticas de riesgo respecto al cuida- do del pie, educación diabetológica previa y control de la diabetes.

- Exploración visual del pié con atención a la higiene, cuidados de las uñas, deformidades, zonas de presión y signos de alteraciones vasculares y neurológicas.

- Exploración neuropática con diapasón de 128 $\mathrm{Hz}$ y monofilamento de Semmes-Weinstein e historial sobre parestesias y dolor neuropático y una exploración vascular con palpación de pulsos e historial de claudicación intermitente.

Se decidió excluir de la exploración vascular el cálculo del índice tobillo-brazo ante el riesgo de obtener datos falsos por las calcificaciones que pueden presentar este tipo de pacientes.

A partir de los datos obtenidos clasificamos a los pacientes según riesgo siguiendo las recomendaciones de la guía de manejo de diabetes basada en la evidencia del Scottish Intercollegiate Guidelines Network ${ }^{7}$.

Se establecieron pues cuatro niveles de riesgo según la existencia o no de alteraciones vasculares, alteraciones de la sensibilidad, deformidades en pies, ulceras previas, déficits visuales y discapacidad física para el autocuidado ${ }^{7}$. A cada nivel de riesgo se le asignó una recomendación sobre el seguimiento que debía tener cada paciente.

El estudio estadístico de los datos fue realizado mediante el programa SPSS 15.0. La asociación de las variables fue evaluada mediante el test exacto de Fisher.

\section{Resultados}

El $77,4 \%$ de los pacientes no había sido explorado nunca para detectar riesgo de pie diabético (tabla 1). El 93,5\% de los pacientes no realizaba controles de hemoglobina glicada (tabla 2). Un 46,7\% presentaba alteraciones en pulsos (tabla 3 ). Un 34,5\% no conservaba la sensibilidad superficial (tabla 4) y un $33,3 \%$ no conservaba la sensibilidad profunda (tabla 5). 
Tabla 1. Existencia o no de exploración previa

\begin{tabular}{|l|l|c|c|}
\hline \multicolumn{2}{|l|}{} & Frecuencia & Porcentaje válido \\
\hline Válidos & Sin exploración previa & 24 & 77,4 \\
\hline & Con exploración previa & 7 & 22,6 \\
\hline & Total & 31 & 100,0 \\
\hline Perdidos & & 1 & \\
\hline Total & 32 & \\
\hline
\end{tabular}

Tabla 2. Realización de controles de Hbalc

\begin{tabular}{|l|l|c|c|}
\hline \multicolumn{2}{|l|}{} & Frecuencia & Porcentaje válido \\
\hline Válidos & Sin controles de Hbalc previos & 29 & 93,5 \\
\hline & Con controles de Hbalc previos & 2 & 6,5 \\
\hline & Total & 31 & 100,0 \\
\hline Perdidos & & 1 & \\
\hline Total & 32 & \\
\hline
\end{tabular}

Tabla 3. Existencia de alteraciones en pulsos

\begin{tabular}{|l|l|c|c|}
\hline \multicolumn{2}{|l|}{} & Frecuencia & Porcentaje válido \\
\hline Válidos & No & 16 & 53,3 \\
\hline & Si & 14 & 46,7 \\
\hline & Total & 30 & 100,0 \\
\hline Perdidos & & 2 & \\
\hline Total & 32 & \\
\hline
\end{tabular}

Tabla 4. Sensibilidad superficial

\begin{tabular}{|l|l|c|c|}
\hline \multicolumn{2}{|l|}{} & Frecuencia & Porcentaje válido \\
\hline Válidos & No conservada & 10 & 34,5 \\
\hline & Conservada & 19 & 65,5 \\
\hline & Total & 29 & 100,0 \\
\hline Perdidos & & 3 & \\
\hline Total & 32 & \\
\hline
\end{tabular}

Tabla 5. Sensibilidad profunda

\begin{tabular}{|l|l|c|c|}
\hline \multicolumn{2}{|l|}{} & Frecuencia & Porcentaje válido \\
\hline Válidos & No conservada & 10 & 33,3 \\
\hline & Conservada & 20 & 66,7 \\
\hline & Total & 30 & 100,0 \\
\hline Perdidos & & 2 & \\
\hline Total & 32 & \\
\hline
\end{tabular}

Tabla 6.Clasificación por grupos de riesgo

\begin{tabular}{|l|l|c|c|c|}
\hline \multicolumn{2}{|l|}{} & Frecuencia & Porcentaje válido & $\begin{array}{c}\text { Porcentaje } \\
\text { acumulado }\end{array}$ \\
\hline Válidos & Bajo riesgo & 7 & 22,6 & 22,6 \\
\hline & Riesgo moderado & 11 & 35,5 & 58,1 \\
\hline & Alto riesgo & 11 & 35,5 & 93,5 \\
\hline & Enfermedad pie diabético & 2 & 6,5 & 100,0 \\
\hline & Total & 31 & 100,0 & \\
\hline Perdidos & & 1 & & \\
\hline Total & 32 & & \\
\hline
\end{tabular}


El $35,5 \%$ de los pacientes presentaba un riesgo alto de lesión, en otro $35,5 \%$ el riesgo era moderado mientras que en un $22,6 \%$ aparecía un bajo riesgo. Sólo en un 6,5\% encontramos el síndrome de pie diabético ya instaurado (tabla 6 ).

No se encontraron diferencias significativas por grupos de edades o sexo.

\section{Discusión}

Numerosos estudios han demostrado que con un plan de prevención sumado a una correcta educación del paciente se pueden reducir un alto porcentaje del número de lesiones sufridas en los pies por los pacientes diabéticos ${ }^{1,2,4}$.

Este planteamiento, aparentemente sencillo, sigue siendo una asignatura pendiente para los profesionales sanitarios que se enfrentan a este tipo de pacientes ${ }^{11,12}$, lo que obliga a hacer una profunda reflexión acerca de los planes de cuidados orientados a este objetivo y a la necesidad de una coordinación interniveles asistenciales. La valoración del pie diabético ha sido destinada a controlar los factores de riesgo para prevenir la aparición de ulceras y a promover el autocuidado. Al realizar esta exploración hemos valorado las aptitudes, habilidades, conocimientos y posibilidades del entorno del paciente y cuidador.

\section{Conclusiones}

A la vista de los resultados obtenidos podemos decir que la ausencia de exploración anterior en la mayoría de los pacientes pone de manifiesto un seguimiento inadecuado y que la alta prevalencia de riesgo de lesión muestra la necesidad de continuar el seguimiento para la prevención de lesiones.

\section{Agradecimientos}

Agradecemos a los miembros del equipo de la unidad de cuidados nefrológicos del Hospital Universitario de Puerto Real que de alguna manera colaboraron en la realización de este estudio y al Dr. Cesar Remón por su asesoramiento en el tratamiento estadístico de los datos.

\section{Bibliografía}

1. Chena JA y colaboradores. Diabetes Mellitus. Aspectos para educadores. Novo Nordisk Pharma, S.A. 2001.

2. Plan Integral de Diabetes de Andalucía 20032007. Disponible en: www.feaed.org/biblioteca/ pdf/diabetesfin.pdf

3. Reparaz Asensio L, Sánchez García-Cervigon P. El pie del diabético. Anales de Medicina Interna (Madrid). [Revista en internet]. 2004, vol. 21, no. 9 [citado 2008-09-20], pp. 5-7. Disponible en: $\quad<$ http://scielo.isciii.es/scielo.php?script=sci arttext\&pid=S0212-71992004000900001\&lng= es\&nrm=iso>. ISSN 0212-7199.

4. Grupo de trabajo Internacional sobre el Pié Diabético. Consenso internacional sobre el Pié Diabético. Madrid. Gramar, A.G. 2001.

5. Papanas N, Liakopoulos V, Maltezos E, Stefanidis I. The diabetic foot in end stage renal disease. Ren Fail. 2007; 29(5): 519-28.

6. Lafuente Robles N, Cruz Arándiga R, Granados Alba A, Batres Sicilia JP, Castilla Romero ML. Guía de atención enfermera a personas con diabetes. ASANEC. Granada. Gráficas Europa, S.C.A. 2003.

7. Management of diabetic foot disease. Scottish Intercollegiate Guidelines Network. Management of Diabetes. 2001: 33-37.

8. Grupo de Trabajo sobre Úlceras Vasculares de la Asociación Española de Enfermería Vascular. Consenso sobre úlceras vasculares y pie diabético. Guía de práctica clínica. Asociación española de enfermería vascular. 2005.

9. American Diabetes Association homepage. Disponible en: http://www.diabetes.org/home.jsp

10. International Diabetes Federation Clinical Guidelines Task Force. Global guideline for Type 2 diabetes. Brussels: International Diabetes Federation, 2005. Disponible en: http://www.idf.org/ home/index.cfm? node $=1457$ 
11. Lancho Casares JM, Juan Larma T, de la Vega Jimenez C, Nieto Guerrero M, Acedo Agúndez J. Paciente en programa de diálisis y arteriopatía periférica. Rev Soc Esp Enferm Nefrol 2008; 11(2): 82-87.

12. Sánchez Perales MC, García Cortes MJ, Borrego Utiel FJ, Viedma G, Gil JM, Pérez del Barrio P, Borrego Hinojosa $J$ et al. Incidencia y factores de riesgo de amputación no traumática de miembros inferiores en los pacientes de hemodiálisis. Nefrología. 2005; 25 (4). 399-406.

13. Lipscombe J, Jassal SV, Bailey S, Bargman JM, Vas S, Oreopoulos DG. Chiropody may prevent amputations in diabetic patients on peritoneal dialysis. Perit Dial Int. 2003 May-Jun; 23(3):255-9.

14. Pliakogiannis $T$, Bailey $S$, Cherukuri $S$, Taskapan $H$, Ahmad M, Oliver T, Bargman JM, Oreopoulos DG. Clin Neprol. 2008 May; 69(5):361-7.

15. Boulton AJ. Lowering the risk of neuropathy, foot ulcers and amputations. Diabet Med. 1998; 15 Suppl 4:S57-9.

16. Locking-Cusolito $H$, Harwood L, Wilson B, Burgess K, Elliot M, Gallo K, Ishe J, Lawrence Murphy JA, Ridley J, Robb M, Taylor C, Tigert J. Prevalence of risk factors predisposing to foot problems in patients in hemodialysis. Neprol Nurs J. 2005 Jul-Aug; 32(4): 373-84.
17. Speckman RA, Frankenfield DL, Roman SH, Eggers PW, Bedinger MR, Rocco MV, McClelIan WM. Diabetes is the strongest risk factor for lower-extremity amputation in new hemodialysis patients. Diabetes care. 2004 Sep; 27(9):2198203.

18. McMurray SD, Johnson G, Davis S, McDougall $K$. Diabetes education and care management significantly improve patient outcomes in the dialysis unit. Am J Kidney Dis. 2002 Sep; 40(3): 566-75.

19. 0'Hare AM, Bacchetti $P$, Segal M, Hsu ChY, Johansen $\mathrm{KL}$. Factors associated with future amputation among patients undergoing hemodialysis: Results from the Dialysis Morbidity and Mortality Study Waves 3 and 4. American journal of kidney diseases. 2003; 41 (1):162-170.

20. ONanda International. Diagnósticos enfermeros: Definiciones y clasificación 2003-2004. Madrid. Elsevier. 2003. 